\title{
Numerical Study of the Effect of Furrows on Biosonar Beamforming in Aselliscus Stoliczkanus Bat
}

\author{
Weikai HE ${ }^{(1),(2),(3)}$, Jianxiong FENG ${ }^{(3),(4)}$, Li GAO ${ }^{(3)}$, Zhiwei ZHANG ${ }^{(5)}$, Hongwang LU ${ }^{(2)}$ \\ (1) School of Life Science, Shandong University \\ Jinan, Shandong Province, China \\ (2) School of Physics, Shandong University \\ Jinan, Shandong Province, China
}

(3) School of Physics and Technology, University of Jinan

Jinan, Shandong Province, China; e-mail: gaoli11281128@sina.com

(4) Department of Mechanical Engineering, Virginia Tech

Blacksburg, VA 24061, U.S.A.

(5) Department of Radiology, Taishan Medical University

Taian, Shandong, China

(received September 28, 2016; accepted April 26, 2017)

\begin{abstract}
The Aselliscus Stoliczkanus bat, studied here, has intricately shaped structures surrounding the nostrils. These structures are hypothesised to have influence on animals' acoustic radiation patterns. Using micro-tomography scanning technique, a 3D digital model of the noseleaf is reconstructed and biosonar beam pattern is analysed using a finite element method based on the $3 \mathrm{D}$ noseleaf model. The present research focuses on the conspicuous furrows in noseleaf, and our analysis allows to conclude the followings: a) structural details in noseleaf of Aselliscus Stoliczkanus bat can produce acoustic effects even if it is not adjacent to the nostrils, b) the furrows possess frequency-selective characteristics, c) the furrows have the function to manipulate the direction and width of the outgoing ultrasound wave.
\end{abstract}

Keywords: Aselliscus Stoliczkanus bat; furrows; frequency-selective characteristics; finite element method; beam pattern.

\section{Introduction}

Bat biosonar can encode information about the presence, location, and even characteristics of sound sources in the environment, like numerous biological hearing systems (Schnitzler et al., 1980; Suga, 1990; Neuweiler, Covey, 2000). The ultrasound emission and reception sites are the only system stages where direction-dependent acoustic diffraction can take place, thus they are critical for this encoding process (MuELLER, 2010). These interfaces form a unique physical substrate for encoding sensory information that is related directly or indirectly to target direction. It is speculated that, due to this pivotal functional role, many species of bats have developed extremely sophisticated vocalisation organs after evolving for a long time. Especially for echolocating bat that emits biosonar pulse through nostrils, complicated structures have been developed around the nostrils (NowAK, 1991; ZhuANG, MuELleR, 2006; 2007). One of the typical and conspicuous facial anatomical structures is the noseleaf, which is used to characterise almost all members of the groups Rhinolophidae and Phyllostomidae (BogdANowicz et al., 1997; VANDERELST et al., 2012). In the anatomic perspective, after a long-term evolution, the noseleaf and outer ear have developed complicated structures that are adaptive to sonar system (Zhuang, Mueller, 2006; 2007; ZHANG et al., 2009; WANG et al., 2010).

It was shown that the surrounding structures of the sound emitting organs have a significant effect on the sonar beampattern. Some numerical evidence so far has emerged to support this notion: sella and anterior leaf of greater horseshoe bats assemble like 
peltate or paraboloid, thus facilitating the concentration of acoustic energy (ZhuANG, MuEller, 2007). It has been shown in literature that when biosonar pulse is at low frequencies, the lancet of the noseleaf acts as resonance cavities that widen the biosonar beam in elevation (GAO et al., 2012). The results from previous study on the acoustic cost function related to sella length of a Bourret's horseshoe bat show that the bat utilises its elongated nose to create a sonar beam with a high focus (ZHANG et al., 2009). Similarly, a change in beam shape was observed when the entire upper portion of a horseshoe bat's noseleaf was covered with petroleum jelly (SCHNitzleR, GRINnell, 1977). Recently, the simulation results of both the hearing and emission subsystems indicate that the noseleaves serve as an energy-focusing part, which mainly performs the task of selective insonification (REIJNIERs et al., 2010; VANDERELST et al., 2010). The above studies show that, for bats that emit sound through nostrils, the structures of noseleaf vary significantly across different species and these different noseleaf structures possess different acoustic properties.

In common with the majority of bats that emit biosonar pulses through their nostrils, Asellicus stoliczkanus bats have noseleaf (Fig. 1), which is one important feature in horseshoe bats. The noseleaf of Asellicus stoliczkanus bat is fairly complex shaped, which is far more complicated than the man-made sound-emission baffles, such as the horns used for megaphones or loudspeakers. The extensive furrow, which is located at the noseleaf upper end, is a noticeable anatomical feature of the bat (Fig. 1). Similar to the furrows in the noseleaf of the greater horseshoe bat (HE et al., 2015), the furrow of Asellicus stoliczkanus is one part of the noseleaf and not adjacent to the nostrils. a)

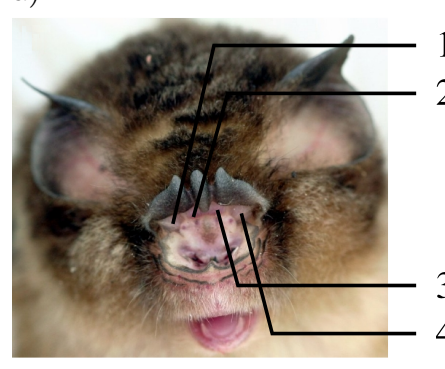

b)

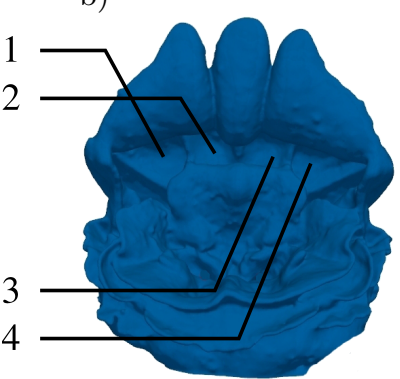

Fig. 1. Shape of the studied noseleaf and its parts: a) portrait photo of the individual of the Aselliscus stoliczkanus bat, b) surface rendering of the noseleaf parts using a smoothed triangular mesh. The digits 1, 2, 3, 4 represent four furrows respectively.

The furrows of Aselliscus Stoliczkanus play an important role in distinguishing them from other species of bats (Koopman, 1994). However, in spite of its use for species classification, the acoustic effect of this structure is waiting to be investigated. It has been widely speculated that the furrows can cause effect on the spatial distribution of acoustic energy emitted by bats. Nevertheless, the experimental data were obtained mainly from simple observation which was not enough to support the hypothesis. In the work reported here, using a numerical approach, we demonstrate the impact of the furrows on the instantaneous near-field pressure magnitude and the radiation patterns.

\section{Experimental setup and methods}

Aselliscus Stoliczkanus is a CF-FM bat, which has frequency range from $104 \mathrm{kHz}$ to $126 \mathrm{kHz}$ (LI et al., 2007). To obtain the 3D noseleaf structural of Aselliscus Stoliczkanus precisely, a high resolution X-ray micro-scanner (Skyscan 1072-Bruker micro-CT, Konitch, Belgium) was used. The scanned images were processed into 3D numerical model, and a numerical method (finite element analysis) is utilised for the simulation of near-field pressure magnitude and the radiation patterns of the bats (Zhuang, Mueller, 2007). In the process of the numerical simulation, the calculation frequency starts from $100 \mathrm{kHz}$ and ends at $130 \mathrm{kHz}$ with the interval as $2 \mathrm{kHz}$.

To investigate the acoustic signals in near field of furrows of Aselliscus Stoliczkanus bat, normalised sound pressure magnitude was obtained in four furrows respectively (ZhuANG, Mueller, 2007). For each frequency, four fixed points are selected out of four furrows, respectively, and each point is treated as the original point. The sound pressure magnitudes of all the data points within the $0.2 \mathrm{~mm}$ radius of the original point is calculated and then averaged. The averaged pressure represents the pressure magnitudes of each furrow (ZHUANG, MUELLER, 2007).

After comparing the sonar beam pattern between natural and filled furrows, it is observed that the furrows have a significant impact on the main and side lobes. To investigate the influence of furrows on the far field, two methods were used:

1. calculating the width of main lobe both in azimuth and elevation that goes through the maximum gain point (ZHANG et al., 2009);

2. calculating the gain of main and side lobes quantitatively (GAO et al., 2011).

In the second method, the beam pattern is divided into main lobe and side lobe areas (GAO et al., 2011).

\section{Results}

The acoustic effect of the furrows of Aselliscus Stoliczkanus bat can be studied by quantitative analysis of the near and far acoustic field, in which the calculation frequency ranges from $100 \mathrm{kHz}$ to $130 \mathrm{kHz}$. 
To analyse the near acoustic field, the pressure magnitudes of both the natural and the filled furrows are calculated (Fig. 2). It can be seen from Fig. 2a that the pressure peak in the natural condition occurs within $116 \mathrm{kHz}$ and $124 \mathrm{kHz}$. In a different frequency range, the sound pressure shows a different tendency: from $100 \mathrm{kHz}$ to $116 \mathrm{kHz}$ and from $121 \mathrm{kHz}$ to $124 \mathrm{kHz}$, sound pressure in all four furrows shows positive correlation with frequency; from $116 \mathrm{kHz}$ to $121 \mathrm{kHz}$ and from $124 \mathrm{kHz}$ to $130 \mathrm{kHz}$, sound pressure in all four furrows shows negative correlation with frequency. The sound pressure distribution for the filled furrows is shown in Fig. 2b. It shows that the sound pressure frequency distribution curve becomes smoother after the furrows are filled, and there is no obvious peak value.
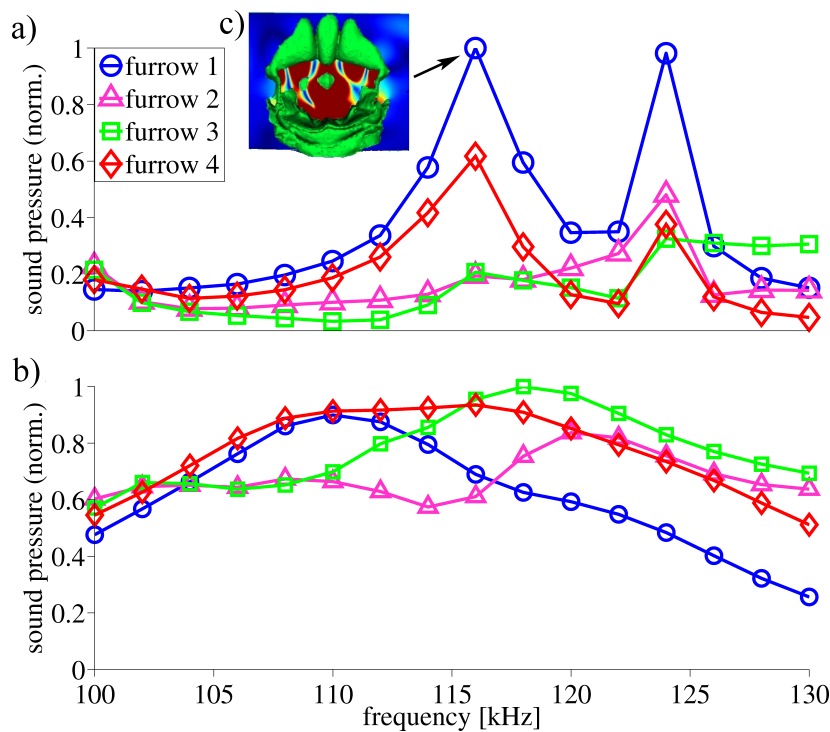

Fig. 2. Normalised sound pressure magnitudes in four furrow as a function of the frequency: a) sound pressure change in four furrows as a function of the frequency for natural furrow, b) sound pressure change in four grooves as a function of the frequency for filled furrow, c) show example the sound pressure distribution in a $2 \mathrm{D}$ section at $116 \mathrm{kHz}$ for natural nostril. furrow 1 , furrow 2 , furrow 3 , furrow 4 represent sound pressure of four furrows respectively.

The effect of the furrows of Aselliscus Stoliczkanus bat on the far field was also investigated. Using the finite element method, the beam patterns with natural and filled furrows were calculated respectively. Comparing the beam patterns between the two conditions (Fig. 3), it can be found that:

1) with the furrows filled, the orientation of the acoustic wave changed dramatically in the frequency range used by bats. For the frequency range from $104 \mathrm{kHz}$ to $126 \mathrm{kHz}$, the main lobe width narrows down both in elevation and in azimuth compared to natural furrows condition;

2) conspicuous side lobes show up after the furrows are filled, while only faint side lobes exist in the natural condition. Besides Aselliscus Stoliczkanus bats, an asymmetric widening of the beam in a sub-band of the biosonar pulse can also be seen in the big brown bat (Eptesicus fuscus), for which the extension of an asymmetric side lobe is controlled by the relative position of the tragus and pinna (MUELLER, 2004).

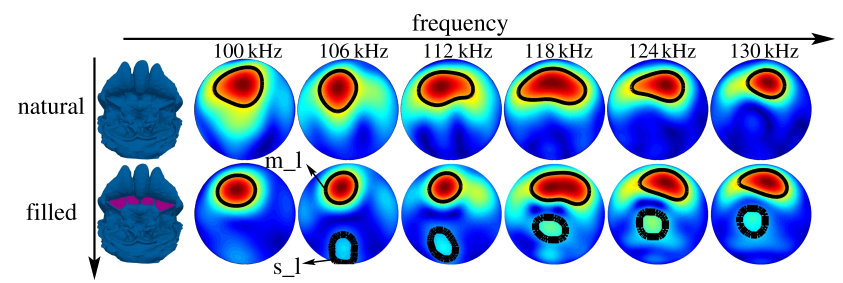

Fig. 3. Numerical beam patterns calculated for different furrow conditions. The first row shows far field beam patterns for the natural furrow. The second row shows far field beam patterns for the filled furrow. Letters m_l are abbreviation for "main lobe", and letters s_l are abbreviations for "side lobe".

The results of the far acoustic field simulation differed pronouncedly between the natural and filled furrow cases. To describe the change quantitatively, main lobe beamwidth both in elevation and azimuth are calculated (Fig. 4). In Fig. 4, for bat's frequency range from $104 \mathrm{kHz}$ to $126 \mathrm{kHz}$, the strong effects of furrows on the ultrasonic beam patterns can be found in terms of beamwidth both in elevation and azimuth. For the natural furrows, the beam pattern contained a single,
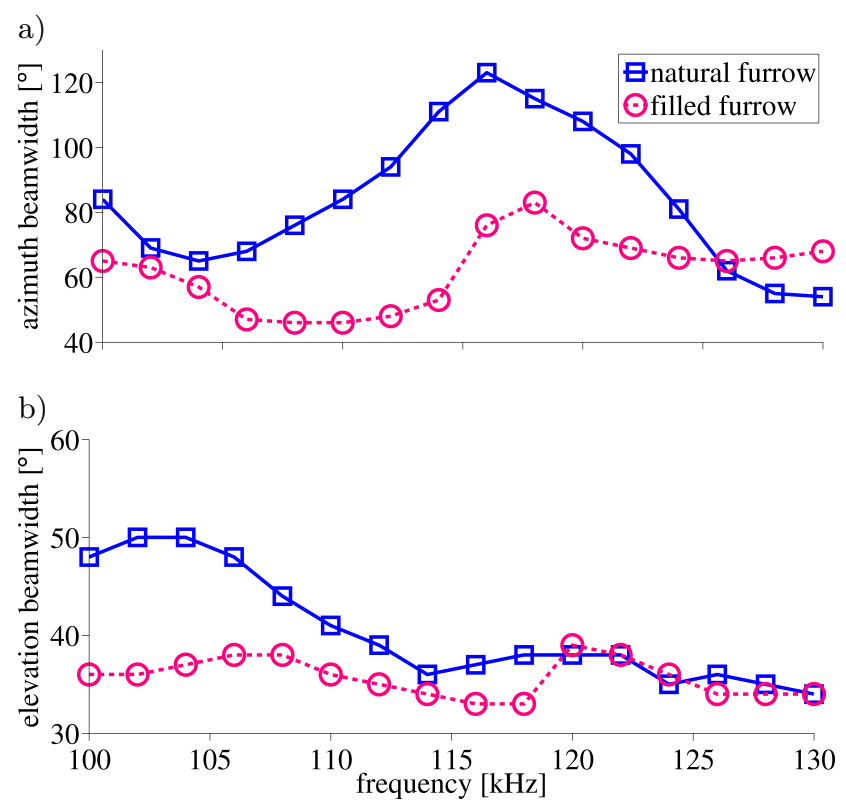

Fig. 4. Azimuth and elevation main lobe beamwidth in natural and filled furrow condition: a) the main lobe's beamwidth in azimuth as a function of the frequency for natural and filled furrow, b) the main lobe's beamwidth in elevation as a function of the frequency for natural and filled furrow. The $x$-axis represents frequency, and the $y$-axis represents main lobe width. 
relatively broad main lobe. After the furrows are filled, the main lobe beamwidth both in azimuth and elevation narrow down itself significantly (Fig. 4). Compared to the natural condition, the beamwidth maximum decline reached $57 \%$ and $28 \%$ in azimuth and elevation, respectively (Fig. 4b, $104 \mathrm{kHz}$ ).

Systematic changes, which are both quantitative and qualitative in the beam patterns can be observed after furrow is filled. For the natural furrow in noseleaf, the side lobes of the beam pattern were much weaker compared to the main lobe (no side lobe in $-3 \mathrm{~dB}$ line, only main lobe, Fig. 3, "natural furrow" case). However, after the furrows were filled, the sensitivity of the side lobes increased significantly (Fig. 3, "filled furrow" case). In this study, the relative contributions of a main lobe to the overall sensitivity of the beam pattern were quantified by integrating the power gain (see Fig. 5). By comparing the proportion of the main lobe energy in total energy between two conditions, it is evident that for the bat's frequency range from $104 \mathrm{kHz}$ to $126 \mathrm{kHz}$, the contribution of the main lobe to the total sensitivity in the normalised beam pattern decreased dramatically after the furrow was filled (Fig. 5). At about $116 \mathrm{kHz}$, the main lobe proportion in the total energy is about two times larger in the natural furrow case than that with the furrow filled, which is the maximum difference for the whole frequency range. The results show that, after the furrow is filled, part of the main lobe energy leaks to the side lobes.

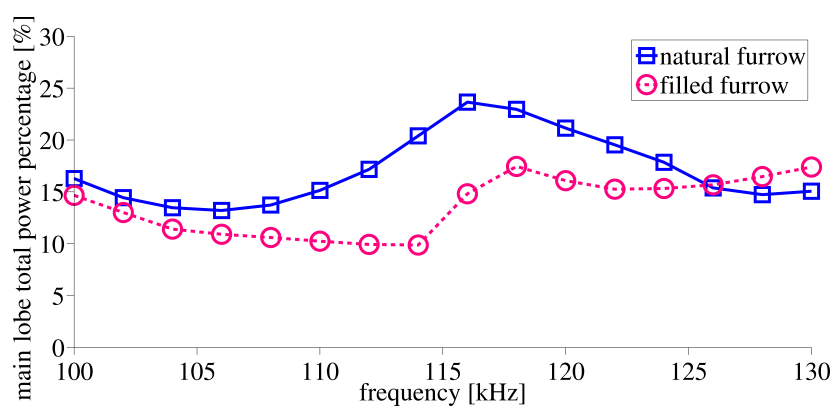

Fig. 5. Main lobe energy proportion in the total energy. The $x$-axis represents the frequency, and the $y$-axis represents the percentage of the main lobe energy. The natural furrow represents the main lobe energy percentage in the natural condition, the filled furrow represents the side lobe energy with furrows filled with voxels.

\section{Conclusion}

By comparing the near field acoustic intensity between the natural and filled furrows, it can be seen that the structure of furrows plays an important role in determining biosonar beam patterns. In the natural furrow case, the pressure distribution of four furrows changes drastically with the increase of frequency with the overall peak value occurring between $116 \mathrm{kHz}$ and $124 \mathrm{kHz}$. However, after the furrows are filled with voxels, the resonance phenomenon disappears (Fig. 2). The spectral and spatial dependence of this effect can be modelled as a resonance cavity (Rossing, FLETCHER, 2004) for which the amplitude is increased only inside the cavity and the resonance occurs at frequencies where reinforcement between the incident and reflected waves takes place. The wavelengths at the experimentally determined resonance frequencies around $116 \mathrm{kHz}(2.9 \mathrm{~mm})$ and $124 \mathrm{kHz}(2.7 \mathrm{~mm})$ are approximately twice as large as the depth (and height) of the furrows (approximately 1.3-1.5 mm). This finding is in fairly good agreement with the resonance frequency of a cylindrical tube, for which one end is open and the other end is closed (Fletcher, 1992). However, the cross-section of the furrows is not circular, thus modelling them as a cylindrical tube can only be the first approximation in spite of the good match of the numbers in this particular case.

The influence of the furrows on the far field is as significant as that on the near field, especially for the main lobe. For the beam patterns of the natural furrow, the main lobe width in elevation and azimuth is larger than that of the filled furrow in bat's frequency range (Fig. 4). The physical underpin that corresponds to this biological behavior is that the enlargement of an aperture relative to the wavelength will narrow the beam it is capable of producing (GoOdman, 2005). In addition, the phenomenon also matches the expectation that the elevation and azimuth dimension confines the degree of the narrowing of the sonar beam, because the filled furrow enlarges the aperture around the nostrils only dorsally but not laterally. This indicates that the furrows have the function of widening the main lobe, thus enlarging the detection area. The main lobe is very helpful in detecting weak signals and locating the acoustic source in the target direction (RAO, BEN-ARIE, 1996). Thus, the furrows play an important role in preying and collision avoidance for Aselliscus Stoliczkanus.

Additionally, with the furrow filled with voxels, the obvious side lobes increased by a large amount and the proportion of the main lobe areas' energy in the total energy decreased as compared to the condition with the natural furrow. The physical phenomenon shows that part of the main lobe energy leaks to the side lobes after the furrow is filled. This shows that the furrows are also capable of concentrating energy, thus enhancing the signal detection sensitivity as well as sound source identification ability.

In conclusion, according to the present research, in the near acoustic field, furrows in Aselliscus Stoliczkanus bat can act as a frequency band filter and energy concentrator in certain frequencies. For the far acoustic field, the transverse furrows have the function of concentrating energy and widening the main lobe. These features are critical in avoiding obstacles and hunting prey for bats. 


\section{Funding}

This work was supported by the National Science Foundation of China under Grant [11404140; 11104355; 61271453], Shandong Province under Grant [BS2013SF001, ZR2016AB13], and University of Jinan under Grant [XBS1332, XBS1610, XKY1637].

\section{Comments}

The first two authors (Weikai He and Jianxiong Feng) contributed equally to this paper.

\section{Acknowledgment}

We would like to thank professor, Dr. Rolf Mueller for his advice and support in this study.

\section{References}

1. Bogdanowicz W., Csada R.D., Fenton M.B.J. (1997), Structure of noseleaf, echolocation, and foraging behavior in the Phyllostomidae (Chiroptera), Journal of Mammalogy, 78, 3, 942-953.

2. Fletcher N.H. (1992), Acoustic systems in biology, Oxford University Press, New York.

3. GaO L., Balakrishnan S., He W., Yan Z., MUELLER R. (2011), Ear deformations give bats a physical mechanism for fast adaptation of ultrasonic beampatterns, Physical Review Letters, 107, 214301.

4. Goodman J.W. (2005), Introduction to Fourier optics, 3rd ed, Roberts and Company Publishers, Greenwood Village, Colorado.

5. He W., Petterson S.C., Gupta A.K., SimMONS J.A., Mueller R. (2015), Lancet dynamics in greater horseshoe bats, Rhinolophus ferrumequinum, PLOS ONE, 10, 4, e0121700.

6. Koopman K.F. (1994), Chiroptera: Systematics, Berlin: de Gruyter.

7. Li G., Liang B., Wang Y.N., Zhao H.B., HelGeN K., Lin L.K., Jones G., Zhang S.Y. (2007), Echolocation calls, diet and phylogenetic relationship of Stoliczka's trident dat Aselliscus stoliczkanus (Hipposideridae), Journal of Mammalogy, 88, 736-744.

8. MÜLLER R. (2004), A numerical study of the role of the tragus in the big brown bat, Journal of the Acoustical Society of America, 116, 3701-3712.

9. MÜLlER R. (2010), Numerical Analysis of Biosonar Beamforming Mechanisms and Strategies in Bats,
Journal of the Acoustical Society of America, 128, $1414-1425$.

10. Neuweiler G., Covey E. (2000), Biology of Bats, Oxford University Press.

11. NowAK R.M. (1991), Walker's Mammals of the World, pp. 169-170, Johns Hopkins University Press, Baltimore, Md.

12. RAo R.K., Ben-Arie J. (1996), Optimal Head Related Transfer Functions for Hearing and Monaural Localization in Elevation: a Signal Processing Design Perspective, IEEE Transactions on Biomedical Engineering, 43, 1093-105.

13. Reijniers J., Vanderelst D., Peremans H. (2010), Morphology-induced information transfer in bat sonar, Physical Review Letters, 105, 148701.

14. Rossing T.D., Fletcher N.H. (2004), Principles of vibration and sound, 2nd Ed, Springer-Verlag, New York.

15. Schnitzler H.U., Henson JR. O.W. (1980), Animal sonar systems, pp. 109-181, Springer.

16. Schnitzler H.U., Grinnell A.D. (1977), Directional sensitivity of echolocation in the Horseshoe Bat, Rhinolophus ferrumequinum. I. Directionality of sound emission, Journal of Comparative Physiology A, 116, 51-61.

17. SugA N. (1990), Biosonar and neural computation in bats, Scientific American, 262, 60-68.

18. Vanderelst D., Mey F.D., Peremans H., GeiPel I., Kalko E., Firzlaff U. (2010), What noseleaves do or FM bats depends on their degree of sensorial specialization, Plos ONE, 5, e11893.

19. Vanderelst D., Reijniers J., Peremans H. (2012), The furrows of rhinolophidae revisited, Journal of the Royal Society Interface, 9, 1100-1103.

20. Wang F., Zhuang Q., Zhang Z. (2010), Frequency Driven Scanning Characteristic of a Pinna Model Inspired from the Brown Big-eared Bat (Plecotus auritus), ACTA Acoustica, 35, 1, 26-30.

21. Zhuang Q., Müller R. (2006), Noseleaf Furrows in a Horseshoe Bat Act as Resonance Cavities Shaping the Biosonar Beam, Physical Review Letters, 97, 21, 218701-1-218701-4.

22. Zhuang Q., Müller R. (2007), Numerical study of the effect of the noseleaf on biosonar beamforming in a horseshoe bat, Physical Review E, 76, 05, 051902-1051902-11.

23. Zhang Z., Nguyen S.T., Müller R. (2009), Acoustic effects accurately predict an extreme case of biological morphology, Physical Review Letters, 103, 038701. 